\title{
The sequence is dead: long live the genome
}

\author{
To what extent do the effort and resources employed in generating a de novo whole genome sequence merit a \\ high-profile publication?
}

This his issue marks the publication of the whole genome sequence of the date palm (Phoenix dactylifera), an economically important crop whose fruit is a staple in many Middle Eastern and North African nations. By facilitating the identification of genome regions specific only to female plants, which produce fruit, the draft sequence promises to yield genetic markers that would provide direct economic benefits to growers. At the same time, the genome provides the first insights into the biology of gender inheritance in this species. And yet some researchers argue that this and other genome papers fail to meet the standards of conceptual novelty and breadth of interest that high-tier journals usually require for publication. As sophisticated sequencing technology becomes increasingly widespread and routine, should high-profile publications turn away more papers describing whole genome sequences? Well, that rather depends.

Many of the first genome publications both celebrated stunning technical achievement and provided significant biological insights. The publication of the first DNA-based genome sequence-that of $\Phi X 174$ bacteriophage-in Nature in 1977 recorded the laborious process of manual sequencing and showed in detail the organization of $\Phi$ X174's tiny DNA complement. The 1990s brought a series of technical advances. Semiautomated sequencers, capillary array electrophoresis and multiparallel detection systems ushered in the era of high-throughput Sanger sequencers, expressed sequence tags and an explosion in microbial genome sequences obtained by shotgun assembly, many of which graced the covers of prominent journals. The most heralded genome of all came in 2001, as the first draft human genome sequences were published in Nature and Science, the former representing the culmination of $\sim 13$ years of effort, $\$ 3$ billion dollars of public funding and the engagement of pretty much all the world's sequencing capacity.

Today, the sequencing landscape looks radically different. It is now routine to sequence a virus or a bacterium, especially if other reference genomes in related genera are available. Whereas ten years ago, there were a handful of sequenced eukaryote genomes (human, yeast, fly, nematode and thale cress) and only 38 prokaryote genomes, the current genome roster comprises 250 eukaryotes, 4,000 bacteria and viruses, and more than 1,000 resequenced human genomes, not to mention the burgeoning number of metagenomes. With the introduction of next-generation instruments, sequence throughput is now a factor of 100 - to 1,000 -fold faster than Sanger technology. An entire human genome can be resequenced in a week, for several thousands of dollars.

Compared with other types of hypothesis-driven biological research, genome papers are sometimes criticized for a lack of intellectual rigor and insufficient biological insight. Genome sequencing, the argument goes, is science by rote-both brute force and high throughput. Even if sequencing an entire genome is not trivial, it remains simply a massive data-generating exercise.

What's more, the data in these papers are already available to the com- munity through GenBank, months or even years before formal publication, albeit without the key quality metrics. As a result, the paper itself can sometimes feel like an afterthought, especially if it merely regurgitates the main statistics and structural features of a genome.

From the perspective of users, the lack of community-approved criteria for assessing the quality, completeness and correctness of a sequence also presents a problem. Understanding the errors in a published sequence governs the types of applications for which it can be used. Thus, a standard draft might be used for mapping traits to regions and serve as reference for RNA sequencing and chromatin immunoprecipitation approaches. But to catalog structural rearrangements and single nucleotide polymorphisms, resolve repeat, homopolymer or GC-rich regions, differentiate pseudogenes from genes, undertake evolutionary comparisons and infer lineage-specific features, a higher quality sequence is needed-checked by Sanger sequencing or long-range, paired-end reads.

For these reasons, it is clear that the days when every bacterial or archeal genome belonged in the pages of a high-tier journal are now gone. The time may also come when the feat of assembling the sequence of a single whole genome is insufficient to merit publication, although the bespoke nature of many current algorithms for next-generation platforms still make de novo genome assembly a nontrivial task.

Genome sequencing is certainly not the billion dollar endeavor it once was. But the publication of a whole genome sequence de novo continues to empower research in scientifically emerging countries, such as Brazil, Singapore and China, spurring international collaborations and consortia. Genome sequencing has provided a starting point to orient and enable hypothesis-driven research, stimulating new lines of investigation years after publication. Above all, it forms the key reference onto which all kinds of data can be layered, including genetic/physical maps, exon sequencing, transcriptomics, proteomics and epigenomics, as well as the basis of inherited disease, cancer, agronomic traits, human ancestry and migration. Ultimately, genome sequence is the digital foundation onto which the systems approaches of the future will be built.

For all these reasons, Nature Biotechnology fully expects to continue publishing whole genome sequence papers. These papers will be of interest to a broad biotech audience because they reveal biologically or commercially relevant insights-insights that could have arisen only from a genome-wide analysis-or they report technical breakthroughs (e.g., the sequencing of complex genomes that are highly polyploid or outbred/heterogeneous in nature). Expect to see more papers that describe not one but many genomes where the value arises from comparisons of different sequences.

In short, neither genomics nor the genome sequence paper is likely to disappear from top journals anytime soon. Imperceptibly, though, the sequence itself is moving away from center stage. And the spotlight is turning on biological insight, biotechnological utility and technological innovation. 